\title{
Cancer screening in the transgender population: a review of current guidelines, best practices, and a proposed care model
}

\author{
Joshua Sterling ${ }^{1}$, Maurice M. Garcia ${ }^{2,3,4}$ \\ ${ }^{1}$ Division of Urology, Rutgers Robert Wood Johnson Medical School, New Brunswick, NJ, USA; ${ }^{2}$ Division of Urology, Cedars-Sinai Medical Center, \\ Los Angeles, Los Angeles, CA, USA; ${ }^{3}$ Department of Urology, University of California San Francisco, San Francisco, CA, USA; ${ }^{4}$ Department of \\ Anatomy, University of California San Francisco, San Francisco, CA, USA \\ Contributions: (I) Conception and design: All authors; (II) Administrative support: MM Garcia; (III) Provision of study materials or patients: J \\ Sterling; (IV) Collection and assembly of data: All authors; (V) Data analysis and interpretation: All authors; (VI) Manuscript writing: All authors; (VII) \\ Final approval of manuscript: All authors. \\ Correspondence to: Maurice M. Garcia, MD, MAS. Director, Cedars-Sinai Transgender Surgery and Health Program; Division of Urology, Cedars- \\ Sinai Medical Center, 8635 W. Third St. Suite 1070 W., Los Angeles, CA 90048, USA. Email: Maurice.Garcia@csmc.edu.
}

\begin{abstract}
Over the last 50 years cancer mortality has decreased, the biggest contributor to this decrease has been the widespread adoption of cancer screening protocols. These guidelines are based on large population studies, which often do not capture the non-gender conforming portion of the population. The aim of this review is to cover current guidelines and practice patterns of cancer screening in transgender patients, and, where evidence-based data is lacking, to draw from cis-gender screening guidelines to suggest best-practice screening approaches for transgender patients. We performed a systematic search of PubMed, Google Scholar and Medline, using all iterations of the follow search terms: transgender, gender non-conforming, gender non-binary, cancer screening, breast cancer, ovarian cancer, uterine cancer, cervical cancer, prostate cancer, colorectal cancer, anal cancer, and all acceptable abbreviations. Given the limited amount of existing literature inclusion was broad. After eliminating duplicates and abstract, all queries yielded 85 unique publications. There are currently very few transgender specific cancer screening recommendations. All the guidelines discussed in this manuscript were designed for cis-gender patients and applied to the transgender community based on small case series. Currently, there is not sufficient to evidence to determine the longterm effects of gender-affirming hormone therapy on an individual's cancer risk. Established guidelines for cisgender individuals and can reasonably followed for transgender patients based on what organs remain in situ. In the future comprehensive cancer screening and prevention initiatives centered on relevant anatomy and high-risk behaviors specific for transgender men and women are needed.
\end{abstract}

Keywords: Cancer screening; trans male; trans female; transgender health; gender affirming hormone therapy

Submitted May 31, 2020. Accepted for publication Sep 04, 2020.

doi: $10.21037 /$ tau-20-954

View this article at: http://dx.doi.org/10.21037/tau-20-954

\section{Introduction}

Widespread cancer screening has resulted in decreased cancer mortality over the past fifty years. Screening has resulted in a $13 \%$ reduction in mortality from colorectal cancer and a $14 \%$ cancer specific mortality in lung cancer $(1,2)$. Mortality rates of breast and cervical cancer have both decreased following widespread adoption of screening mammograms and pap smears $(3,4)$. PSA screening does reduce prostate cancer mortality but is also associated with false-positive and overtreatment $(5,6)$. The American Cancer Society (ACS) and US Preventative Services Task Force (USPSTF) and numerous professional organizations (ACS, AMA, AUA, ACOG) have clear recommendations for the early detection of cancer in average-risk and high- 
risk cis-gender patients.

These guidelines become less straight forward when applied to the transgender community and currently World Profession Association of Transgender Health (WPATH) has no guidelines on cancer screening. It is important to consider that transgender patients' cancer screening needs will vary by "what stage of their transition" they are in as initiation of gender-affirming hormone therapy (GAHT), non-genital gender affirming surgery (GAS), genital GAS, and surgical removal of some or all of their reproductive organs may affect cancer risk. WPATH Standards of care version 7 states, "In the absence of large-scale prospective studies, providers are unlikely to have enough evidence to determine the appropriate type and frequency of screening...Patients may find cancer screening gender affirming, or both physically and emotionally painful" (7). Large databases in the United States, like the Surveillance, Epidemiology and End Result (SEER) and the National Cancer Database (NCDB), do not capture non-binary genders, thus it is difficult to postulate if the cancer risk of transgender individuals is different from the general population. A UK study found gay and bisexual men had increased odds of a cancer diagnosis compared to heterosexual males; although main driver of this difference was the higher rates of viral-related cancers: Kaposi's sarcoma, anal cancer, and penile cancer (8). Studies in the US have tried to look at cancer rates in areas with a high population of LGBT individuals to extrapolate any associated cancer risk $(9,10)$; the results of these studies were varied and no firm conclusions can be drawn from them. The differences in cancer rates seen in the LGBT community is often attributed to high risk behaviors: smoking, alcohol and drug use, obesity, and significantly higher HIV rates (11-13). The CDC reported that in 2013, $1.9 \%$ of HIV tests done by transgender individuals were positive, compared to $0.9 \%$ for cis-gender males and $0.2 \%$ for cis-gender females (14). The estimated prevalence of $\mathrm{HIV}$ among transgender women of reproductive age (range, $15-49$ ) is $21.7 \%$ (95\% CI: $18.4-25.1 \%$ ), which is 34 times higher than cis-gender adults in the same age range (15).

Transgender patients often face discrimination and are stigmatized in ways that decrease healthcare screening encounters. Transgender individuals have reported difficulties when interfacing with the US healthcare system: $19 \%$ have reported refusal of care, $28 \%$ reported harassment, and $50 \%$ were turned off of the healthcare system due to a lack of gender nonconforming providers
$(16,17)$. Clinicians also may fail to provide the appropriate screening and counseling based on the patient's anatomy. This includes PSA checks and prostate exams for anyone that still has a prostate and Pap smears for patients that still have a cervix, regardless of what gender they identify with.

Additionally, many transgender patients only seek medical care as a part of gender affirmation and may avoid primary health care concerns. Patients that do seek routine healthcare checkups are reluctant to bring up gender incongruous organs. Hence, transgender patients may be more reliant on their health care providers to initiate cancer screening discussions than cis-gender patients.

The aim of this review is to cover current guidelines and practice patterns of cancer screening in transgender patients, and, where evidence-based data is lacking, to draw from cis-gender screening guidelines to suggest bestpractice screening approaches for transgender patients.

We performed a systematic search of PubMed, Google Scholar and Medline, using all iterations of the follow search terms: transgender, gender non-conforming, gender nonbinary, cancer screening, breast cancer, ovarian cancer, uterine cancer, cervical cancer, prostate cancer, colorectal cancer, anal cancer, and all acceptable abbreviations. Given the limited amount of existing literature inclusion was broad. After eliminating duplicates and abstract, all queries yielded 85 unique publications. We present the following article in accordance with the PRISMA-ScR reporting checklist (available at http://dx.doi.org/10.21037/tau-20-954).

\section{Cancer screening guidelines}

It has been shown that early detection of cancer results in improved outcomes $(1,3,4)$. However, when developing screening guidelines, the benefit of early detection must be balanced with the fact that most people screened will receive no benefit and could potentially be suffer harm. Finding that balance is the reason that guidelines vary in testing frequency and strength of recommendation between different professional organizations. Due to this uncertainty and the inherent risks of these screening procedures open and frequent dialogue between patient and physician is very important in determining what screening protocol is best for an individual.

Large population data sets like, SEER, are used to identify high risk population that would benefit most from screening procedures. Transgender and non- 
binary individuals are poorly captured in these databases making comparisons between the transgender and general populations and identification of high-risk sub-populations within the transgender community very difficult $(7,18)$. There are currently very few transgender specific cancer screening recommendations. All the guidelines discussed in this manuscript were designed for cis-gender patients and applied to the transgender community based on small case series.

\section{Youth cancer risk}

Gender dysphoria does not affect a patient's cancer risk, but it does decrease a patient's chance of undergoing screening for their birth sex organs owing to provider factors (lack of education and comfort) and patient factors (aggravation of gender dysphoria with physical examination of sex organs) (19). Children as young as 2 years old may show signs of gender dysphoria; for most children gender dysphoria will disappear before the onset of puberty (20). Internal survey of our patients found $71 \%$ experienced their first experiences of gender dysphoria in early childhood, between the ages of 3-6. A patient's baseline dysphoria is often exacerbated by interactions with the medical community which often result in patients being forced to acknowledge and undergo examinations of birth sex organs. According to transgender patients when they do have doctor visits the focus is on GAHT or mental health concerns, with physical ailments being the least important concern (21). A review of the literature regarding primary care in transgender patients reinforces these priorities; general health, including cancer, is the least researched aspect of the transgender global burden of disease initiative (22). The lack of focus on primary health concerns, both by transgender patients and physicians, decreases the likelihood that they will have any sort of cancer screening. The discrimination transgender patients experience when interfacing with the health care system does not easily foster the kind of open conversations needed to discuss a topic like cancer screening, given the lack of clear evidence and professional guidelines.

GAHT has been shown to be safe and have predictable effects on hormone levels in transgender youths (23). While an increased cancer risk with the use of hormone therapy is theoretically possible and plausible, there have been no well-designed studies published showing that long term use of GAHT or suppression of a patient's natal puberty increases cancer risk (24). However, the uncertainty and possible risk is frequently emphasized in discussion between patients and transgender patients without placing an equal consideration on the benefits of GAHT (25).

\section{Social transition and cancer risk}

A patient's stage of gender transition can affect the type of medical specialist they see most regularly, which in turn can alter which cancer screening protocols are emphasized. Transgender patients report to us that they experience a sense of unease with seeking birth-sex based cancer screening during and after gender transition as doing so requires acknowledging that they retain birth-sex anatomy. For example, transmasculine men with ongoing gynecologic cancer risk factors may be less likely to follow-up with a general gynecologist after starting GAHT and/or genital GAS. For those patients who have already transitioned socially going to a gynecologist can be very difficult; it can be seen as a step backwards for transmasculine patients, and transfeminine patients may find that they are turned away. Again, examinations of a patient's birth sex organs are very likely to aggravate any gender dysphoria and need to be done with extra care and consideration for this fact. In 2011, ACOG released a statement that healthcare providers should be able to care for transgender individuals or at least be able to refer them for routine checkups and screening as appropriate (26).

\section{Gender affirming hormone therapy and cancer risk}

The long-term effects of GAHT is a potential challenge unique to transgender patients. GAHT for transgender people is different from hormone replacement therapy for cis-gender people in two ways: (I) when GAHT is provided before surgical removal of their birth-sex gonads, the patient may have elevated serum levels of both masculinizing and femininizing hormones; (II) the effective dose of GAHT can vary widely by individual patient, such that some have significantly high serum levels of a particular hormone or its metabolites, which can increase (or decrease) risk with sex hormone-sensitive cancers.

The administration of sex hormones not only makes the interplay between birth-sex and cancer risk more complicated as they may not only affect the risk of sexspecific cancers but also for other cancers and/or organ systems (e.g., cardiovascular) that contain sex-receptors. 
The role of estrogens and androgens in breast and prostate cancer has been well studied in cis-gender individuals and is emerging as an active area of transgender research. The presence of estrogen and progesterone receptors is a major factor in the prognosis and treatment of cis-females and cis-males with breast cancer (27). Androgen receptors are present in most breast cancers and can have tumor suppressive or tumor proliferative effects depending on the type of breast cancer (28). In all of these instances the effect of exogenous hormones on cancer risk or cancer treatment is still unknown (29).

The effect of exogenous hormones has not been investigated in the US to date but there have been published studies from the Netherlands (30,31). One study looked at over 2,300 transgender patients between 1975 and 2011 who all received GAHT. Over that time two breast cancer cases were reported in the transfeminine population, one case of breast cancer was reported in the transmasculine population, and one case of prostate cancer was reported. These rates were lower than breast cancer rates and prostate cancer rate for cis-females and cis-males respectively $(30,31)$.

The WPATH SOCv 7 states that the current evidence is insufficient to determine if feminizing hormones increase a patients risk of breast cancer but notes that risk of breast cancer in transfemales is not zero (7). There is no evidence masculinizing hormones increases a patient's risk of breast or cervical, although it may increase the incidence of abnormal pap smears (7). The current evidence is inconclusive to determine how masculinizing hormones affect the risk of ovarian, or uterine cancer (7).

\section{General transgender screening recommendations and considerations}

There are currently no established cancer screening guidelines, recommendations, or protocols for transgender patients at any point in their transition. The recommendations presented in this manuscript are based on accepted guidelines for cis-gender patients and applied to the transgender community based on small case series. The screening protocols that will be discussed in this manuscript should not just be applied to any patient without a full discussion with the patient about where they are in their transition, what natal organs are still present, and what other lifestyle factors would make the patient high risk. These recommendations are intended to foster open dialogue between patients and physicians about what cancer screening they may benefit from given the overall lack of large-scale prospective data to guide these decisions.

\section{Colon cancer}

Patients over 50 should be screened for colorectal cancer with either guaiac-based fecal occult blood test, fecal immunochemical test, multitarget stool DNA test, doublecontrast barium enema or CT colonography (6). The frequency of screening depends on the screening method and any positive test needs to be followed up with a colonoscopy. Patient's considered to be high risk, due to personal history of disease, family history or history of inflammatory bowel disease are recommended to undergo more intensive surveillance regimens (6). No publications were found that examined if rates of colorectal cancer in transgender patients differed from the general population. These guidelines should also be followed for all transgender individuals at any point in their transition.

\section{Lung cancer}

Current or former smokers of either sex with 30 pack year history, or former smokers who quit less than 15 years ago should discuss annual lung cancer screening with their health care provider (6). No publications were found that examined if rates of lung cancer in transgender patients differed from the general population. These guidelines should also be followed for all transgender individuals at any point in their transition.

\section{Anal cancer}

There is not a specific guideline about screening for anal cancer, but the American Society of Clinical Oncology recommends that routine testing for high risk patients, defined as HIV-infected individuals that engage in anal receptive intercourse. The prevalence of HPV and rate of $\mathrm{HPV}$ vaccination in the transgender community is poorly reported. What data is available groups all LGBT individuals into a single category which does not provide any granularity or useful specificity. HPV is known risk factor for developing anal cancer especially in persons that engage in anal receptive intercourse. Many younger patients are unaware of this risk and not being accustomed 
to needing medical screening do not ask physicians to be checked; additionally, physicians are often reluctant to bring up HPV or perform routine inspection of the anus (32).

The most common screening method is cytology from an anal pap smear and confirmatory testing is done with anoscopic biopsy. Similar to Pap smear, cytological screening aims to detect anal high-grade squamous intraepithelial lesions (HSIL), and as with cervical washings these are considered pre-malignant with the potential to progress to anal cancer (33). A 2017 study of HIV positive transfeminine patients found that $91 \%$ of patients biopsied had some degree of dysplasia, although none of the patients screened were found to have cancer (33). Another study out of Thailand found that $42 \%$ of transfeminine patients screened had abnormal cytology (34). There is evidence that transfeminine patients, especially $\mathrm{HIV}+$ patients, are at increased risk on anal squamous intraepithelial lesions thus, there is a clear need for a standardized, evidence-based screening process. While there is no consensus Thompson et al. proposes screening any transfeminine patients with multiple lifetime sexual partners starting at age 21 (35). Transmasculine patients that engage in anal intercourse should discuss screening options and frequency with their physician. These guidelines should also be followed for at any point in during a patient's transition.

\section{Sex organ specific screening recommendations: transmasculine patients}

With GAHT, transmasculine individuals, are often administered only exogenous testosterone. There is little evidence that testosterone increases the incidence of cancer in female reproductive organs. Olsen et al. found cisgender females that used testosterone supplements had an increased risk of ovarian cancer, but it is not clear how this data translates to the transmasculine population as follow up in transmasculine patients who still have their natal reproductive organs is small (36). The largest such study performed histologic analysis of specimens from 112 patients who underwent at least 6 months of GAHT prior to total abdominal hysterectomy bilateral salphingo-oopherectomy. There weren't any cases of ovarian, uterine, or cervical cancer, and no observed pre-malignant changes leading the author to conclude GAHT did not pose an increase ed cancer risk (37). There have been reported cases of transmasculine patients with female reproductive organ malignancies: six cases of ovarian cancer, three cases of cervical cancer, one case of vaginal cancer and one case of uterine cancer.

\section{Breast cancer}

Recommendation for cis-females is they should be screened for breast cancer with annual or biennial mammograms after 50; screening can start earlier based on family history or patient preference. As previously stated, research as shown that transmasculine patients on GAHT do not have an increased risk of breast cancer. In fact, early reports suggest that their risk is similar to that of cismales $(30,38,39)$. Brown et al., using data from the Veteran Health Administration, found seven cases of breast cancer in transmasculine patients, and $52 \%$ of patients had undergone some GAHT treatment. The overall incidence was 20/100,000 patient years regardless of hormone exposure, which was not higher than the expected rate (39). Four cases of invasive breast cancer were found in 1,229 transmasculine patients, which was lower than expected compared to cisgender women (incidence ratio 0.2, 95\% CI: 0.1-0.5) (40).

Breast cancer screening guidelines are an evolving area of medicine, with respect to what age to begin screening and frequency, which is made more complicated in transmasculine patients by a poor understanding of the effect of GAHT and the lack of reliable epidemiologic data. Transmasculine patients who have not undergone bilateral mastectomy or who have only undergone breast reduction should follow screening guidelines for cis-females. There is currently no reliable evidence to guide the screening of transmasculine patients after mastectomy. Annual chest wall exams, ultrasound and MRI have all been suggested but further research is needed before any recommendations for screening in this patient population can be considered evidence based (41-44). The best recommendation for transmasculine patients at any point in their transition is, "Screen often and screen what you have" (43).

\section{Uterine cancer}

There is currently insufficient evidence to support screening cisgender females for uterine or endometrial cancer. A 2015 survey of transmasculine patients found only $8 \%$ of respondents have their uterus and cervix removed (45). Patients that still have a uterus should inform their physician of any abnormal vaginal bleeding. The reported case of uterine cancer was discovered during pre- 
operative workup for genital gender affirmation surgery, when the patient who had been amenorrhoeic for seven years had vaginal bleeding (46). Screening or prophylactic hysterectomy for uterine or endometrial cancer are not recommended but endometrial evaluation is recommended as a part of genital gender-affirmation surgery $(46,47)$.

\section{Cervical cancer}

Screening guidelines in cis-females are: patients over 21 should undergo cervical cancer screening with pap smears and after the age of 30 pap smears should be accompanied by HPV DNA tests (6). Screening should continue until the patient no longer has a cervix or the patient is over 66 with 2 consecutive negative tests.

Compared to cis-females, 9.2\% fewer transmasculine patients were up to date on their cervical cancer screening $(48,49)$. The use of GAHT does not increase risk of cervical cancer, however transmasculine patients do have lifestyle factors which may increase their risk. Rates of active HIV in the transgender population are $2 \times$ higher compared to cis-males and $10 \times$ higher compared to cis-females another factor confounding cervical cancer risk in transmasculine patients $(14,15,50)$. The strong association between cervical cancer and several types HPV may increase the overall cancer risk in transgender patients because HIV infection increases the likelihood of a persistent HPV infection. More specific research is needed to understand the impact of HPV and associated cancers on the overall health outcomes of transgender patients $(29,51,52)$. GAHT will cause the cervical epithelium to atrophy and thus transmasculine patients have a 10 -fold increased likelihood of an inadequate test compared to cisgender females (53). Other factors associated with inadequate pap smear were higher body mass index and longer testosterone use (53). After an inadequate test patients were less likely to return for retesting in one year, overall it took transmasculine individuals 5 times longer to return for retesting compared to cis-females, which can explain why transmasculine patients are less likely to be compliant with screening guidelines (52). Suggested provider techniques to optimize adequate cervical sampling include swabbing a wide circumference, using multiple sampling tools and use of low-dose topical estrogen for five nights prior to examination $(54,55)$.

Annual pap smear is recommended for transmasculine patients over 21 if the cervix is present (56).

\section{Ovarian cancer}

The USPSTF currently recommends against routine screening of cisgender women for ovarian cancer (57). A review of the literature found no strong evidence that transmasculine patients are at increased risk of ovarian cancer $(36,58)$. It is recommended that transmales follow the guidelines for cis-females, routine cancer screening is not recommended, and prophylactic oophorectomy without other risk factors is unnecessary (58).

\section{Vulva cancer}

There is currently insufficient evidence to support screening cisgender women for vulvar cancer. Diagnosis is made with physical exam and confirmed with tissue biopsy. Based on the solitary case of vaginal cancer in the literature there is no recommendation for routine screening tests in low or high-risk patients at any point in their transition.

\section{Post gender affirming surgery considerations}

As previously stated, there is no data on breast cancer after gender affirming mastectomy; annual chest wall exams, ultrasound and MRI have all been suggested, but further research is needed before any recommendations can be made. Surgeons should be aware of the malignancy potential of organs removed during gender affirming surgery and should always review all pathology reports. Physicians should also be cognizant of and note any remaining portions of birth sex organs left behind after gender affirming surgeries so that potential differential diagnoses are not missed.

\section{Sex organ specific screening recommendations: transfeminine patients}

GAHT in transfeminine patients can include GnRH antagonists to shut down an individual's natal sex hormone production in addition to the administration of exogenous estrogen. The administration of these hormones makes the complex interactions between sex and cancer risk more complicated as they may not only affect the risk of sexspecific cancers but also for other cancers that may contain sex-receptors. As with the female sex organs there is no evidence that GAHT increases oncologic risk of the male sex organs. 


\section{Testicular cancer}

Cis-males should have annual physical examinations to monitor for any testicular masses. There have been two reported cases of testicular cancer in transfeminine patients: one was found incidentally after orchiectomy and the other was discovered when the patient had rising testosterone levels despite being on feminizing hormones $(59,60)$. It is recommended that patients that still have testicles undergo annual physical exam to check for any testicular masses at any point in their transition.

\section{Prostate cancer}

Prostate cancer screening recommendation for cis-males is patients over 50 have their PSA checked and digital rectal examination. This screening should occur earlier in high risk patient: African-Americans and patients with a positive family history. The risk of prostate cancer in transfeminine patients is lower than cis-males but it is not zero.

Even though the prostate is not removed during surgical transition, there are few studies looking at the prevalence of prostate cancer in the transfeminine population. Gooren et al. reported a prevalence of $0.04 \%$ in their cohort of over 2,300 patients, however these patients were not screened for prostate cancer and the actual prevalence maybe higher (31). This lack of screening for prostate cancer has been shown in other studies too. Tabaac et al. also found that transfeminine patients were less likely to discuss prostate issues with a physician and were less likely to have had a PSA test compared to cis-males (16). This may have been considered a nonissue in transfeminine patients because "dissemination of prostate cancer is inhibited by eliminating androgens or neutralizing their effect with the injection of estrogens" (61). More recent studies and case reports, including the 10 cases of prostate cancer in transfeminine patients of which 6 were metastatic on presentation, are beginning to question this dogma. The patients in all reported cases of prostate cancer had undergone extended estrogen therapy and some studies have suggested that estrogen may play a role prostate cancer development (62-64). Although none of these studies have been conducted in the transgender population. Both the WPATH and the Endocrine society recommend transfeminine patients follow the current prostate cancer screening guidelines for cis-men with the caveat that $1 \mathrm{ng} /$ $\mathrm{mL}$ should be considered the upper threshold of normal $(56,65,66)$. This guideline should be followed at any point in a patient's transition.

\section{Penile cancer}

There is currently insufficient evidence to support cancer screening for penile cancer in the cis-gender population. Diagnosis is made based on physical exam and confirmed with a biopsy. There have been no reported cases of penile cancer in the transgender population. However, patients that still have a penis should report any lesions to their physician.

\section{Breast cancer}

There is not sufficient evidence to support breast cancer screening in cis-males and as previously stated cis-females should be screening with routine mammograms. Brown et al., using data from the Veteran Health Administration, found three cases of breast cancer in transfeminine patients, most patients had undergone some GAHT. The overall incidence was 20/100,000 patient years regardless of hormone exposure, which was not higher than the expected rate (39). De Blok et al. found 15 cases of breast cancer out of 2,260 transfeminine patients, and one study from the Netherlands found transfeminine patients have a 46 -fold increased risk of breast cancer compared to cisgender men (40). Most tumors presented in a typically female pattern, they were ductal carcinomas and receptor positive. It should be noted that the rate of breast cancer in transfeminine individuals was still lower than cisgender females (incidence ratio of 0.3 ) (40).

Transfeminine patients have an overall lower exposure to estrogen and little exposure to progesterone compared to cisgender females, which could explain their lower cancer risk. The presence of estrogen and progesterone receptors is a major factor in the prognosis and treatment of cisfemales and cis-males with breast cancer (27). Androgen receptors can have tumor suppressive or tumor proliferative effects depending on the type of breast cancer, and while there isn't evidence to suggest GAHT increases cancer risk the effect of exogenous hormones on cancer risk or cancer treatment is still unknown $(28,29)$. There is also evidence to suggest testosterone is protective and the loss of circulating testosterone combined with the increased circulating estrogen levels results in the increased risk for transfeminine patients compared to cismales (28). Transfeminine patients commonly have dense breasts, which is both an independent risk factor for breast cancer and increases the rate of falsenegative mammograms.

There is no current data or recommendations on how 
GAHT affects breast cancer risk for patients with BRAC1 mutations, only $6 \%$ of cisgender men with BRAC1 mutation develop breast cancer compared to $78 \%$ of cisgender females (41). There has been one case report of a transgender female with a BRCA-1 mutation. This patient refused prophylactic mastectomy, continued GAHT after her vaginoplasty and bilateral orchiectomy, and is undergoing screening based on the established guidelines for cisgender women (67).

Breast cancer screening guidelines are an evolving area of medicine, with respect to what age to begin screening and frequency, which is made more complicated in transgender women by a poor understanding of the effect of GAHT and the lack of reliable epidemiologic data. However, for transfeminine patients' mammograms are recommended every two years in patients over 50 and who have $5-10$ years of GAHT treatments (41). All screening should be based on shared decision making, and patients and providers may want to start screening at an earlier age or shorter number of years of hormone exposure in patients with a significant family history.

\section{Malignancies of the neovagina}

There are several techniques and approaches used to create a neovagina all of which have the potential to develop malignancy; the use of genital skin flaps is the gold standard for genital affirmation surgery and intestinal transplant vaginoplasty is more commonly used in patients with congenital or traumatic absence of the vagina. Skin transplants developed squamous cell carcinoma (SCC) of the neovagina while intestinal neovaginas developed adenocarcinoma. There are 4 reported cases of neovaginal SCC in transfeminine patients and 3 of the 4 reported cases also had HPV (68). The median age of vaginoplasty was 27 and the latency period prior to diagnosis ranged from 18 to 45 years with a median latency period of 26 years (68-71). There have been 7 reported cases of neovagina adenocarcinoma following colon transplantation and 1 case with a small intestine transplant; none of these cases were in transgender individuals $(72,73)$. Secondary malignancy at the ureteroenteric anastomosis is a well-documented long-term complication of ureterosigmoidostomy and it has been proposed that chemical stimulants and irritants in semen contribute to malignancies of the neovagina. Any association between HPV and adenocarcinoma occurring in an intestinal transposed neovagina is still unknown. Grosse et al. looked at cytology from neovagina washing from 20 patients, three colon transplants and 17 skin grafts, and found $30 \%$ had abnormal cytology. The authors concluded that patients with neovaginas, regardless of graph tissue, were prone to precancerous lesions, and advised patients engage in cancer screening programs (74). Although the optimal follow up protocol has yet to be defined.

This is a rare complication following vaginoplasty, but physicians must be aware of the persistent risk of malignant transformation. There are no established guidelines the Endocrinology society suggest routine cancer screening for transfeminine patients. Screening should include annual postoperative physical exam including speculum and digital neovaginal examination and cytologic testing every 3 years starting at 21 until 70 (56). HPV vaccination is recommended for all transgender patients under age 26.

\section{Post gender affirming surgery considerations}

As previously stated, surgeons should be aware of the malignancy potential of organs removed during gender affirming surgery and should always review all pathology reports. Physicians should also be cognizant of and document any birth sex organs left behind after gender affirming surgeries.

\section{Best practice tables}

Summary of cancer screening recommendations for transmale and transfemale patients (Table 1).

\section{The biopsychosocial model and transgender healthcare}

When counseling patients' physicians often rely on the biomedical model, which has been the dominant model for Western medicine since the 19th century. The biomedical model focuses on health status, and achieving freedom from compromised health. By focusing on decreasing chronic conditions, it creates a common language and understanding between the physician and patient. Such commonality of focus and language is harder for many transgender patients because of the fact that many patients have very personalized transition goals, and, due to the lack of information regarding long-term outcomes in transgender patients. Other important factors that limit the utility of the biomedical model for transgender care is because some care providers lack of understanding about not only what it is to be transgender, but also, how being transgender can affect- 
Table 1 Best practices cancer screening protocol

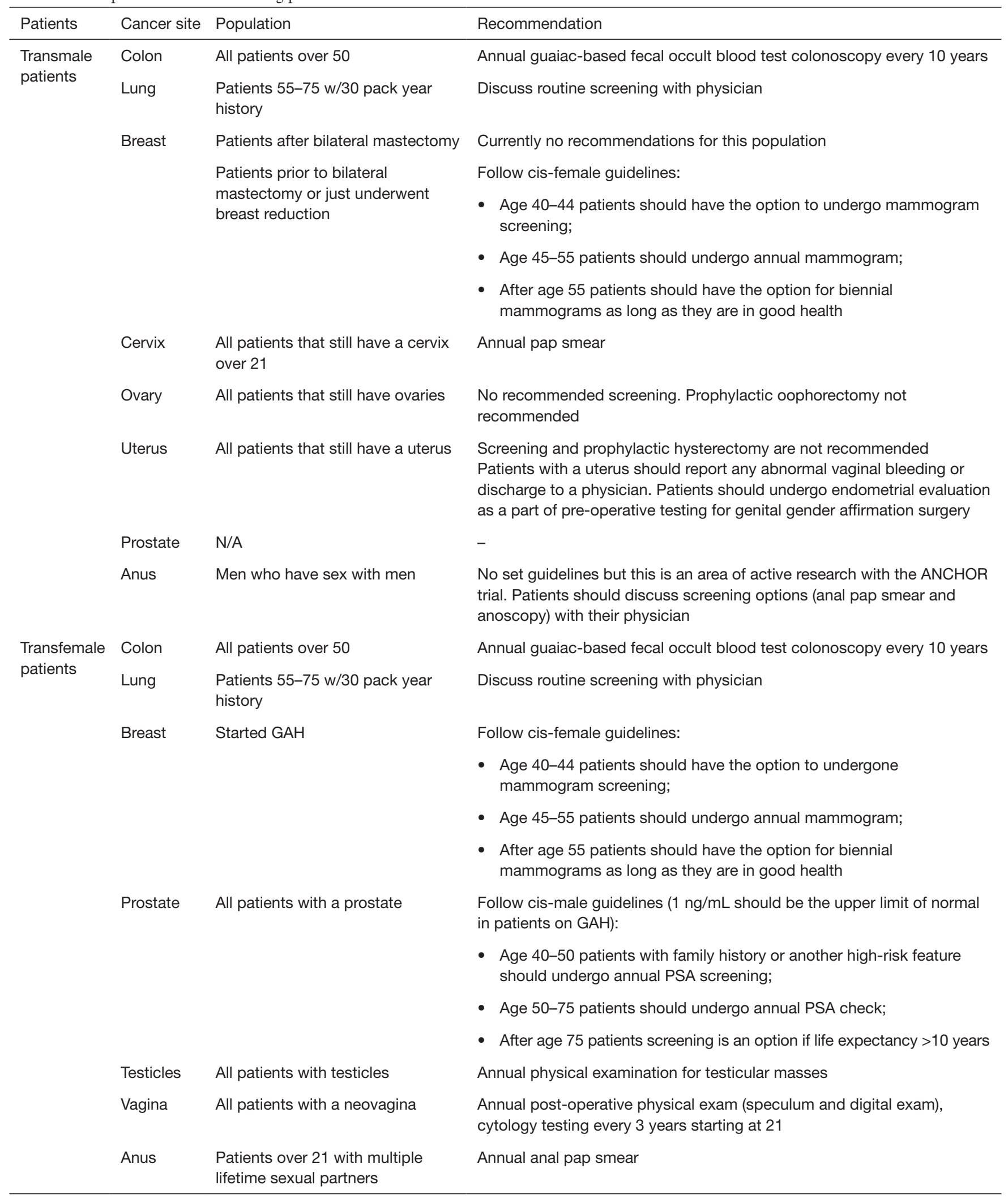

HPV vaccination is recommended for all transgender individuals through age 26 . 


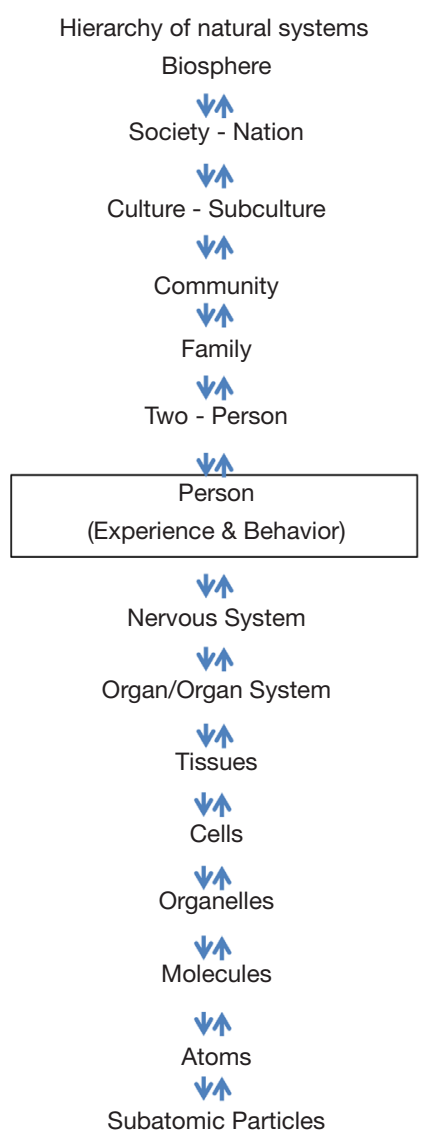

Figure 1 Hierarchy of natural systems.

and be affected by, different aspects of the patient's' social world and health. The disconnect that results can for many patients foster distrust towards the healthcare system.

We propose a new model for transgender healthcare, which is rooted in the Biopsychosocial Model first proposed by George L. Engel and Jon Romano in 1977. Engel and Romano's model focuses on the development of illness from the complex interactions across and within biological, psychological, and social systems (Figure 1) $(75,76)$. Engel emphasized that the biomedical approach is flawed because the body is not the only contributor to illness, or wellness $(76,77)$. Instead, an individual's own psychological (mood, personality, behavior, etc.) and social (cultural, familial, socioeconomic, etc.) domains also significantly impact underlying biological (genetic, biochemical, etc.) factors, to determine how illness and health are caused and treated (75). Engel also emphasized the need for two-way dialogue between the patient and doctor in order to find the most effective treatments (76).
We note that the process of gender transition affects (and is affected by) both biological and social continua. For example, gender affirming hormone therapy and surgery are a part of gender transition for many transgender/ gender non-conforming people, as is a significant change in an individual's gender and social roles. A common theme in the World Professional Association for Transgender Health (WPATH) Standards of Care (SOC) Guidelines is that a cornerstone of care for the transgender patient is to facilitate and adapt to positive change in mental health, social domains, and for some, physical/body related domains (7).

What is perhaps less obvious from the biopsychosocial model is that for people undergoing gender transition, certain subdomains of the biological and social continua change significantly, and often over a relatively short period of time. It is useful for healthcare providers to consider how changes in sex hormones, body appearance, dress, personal pronouns, partner, family, and professional relations can 


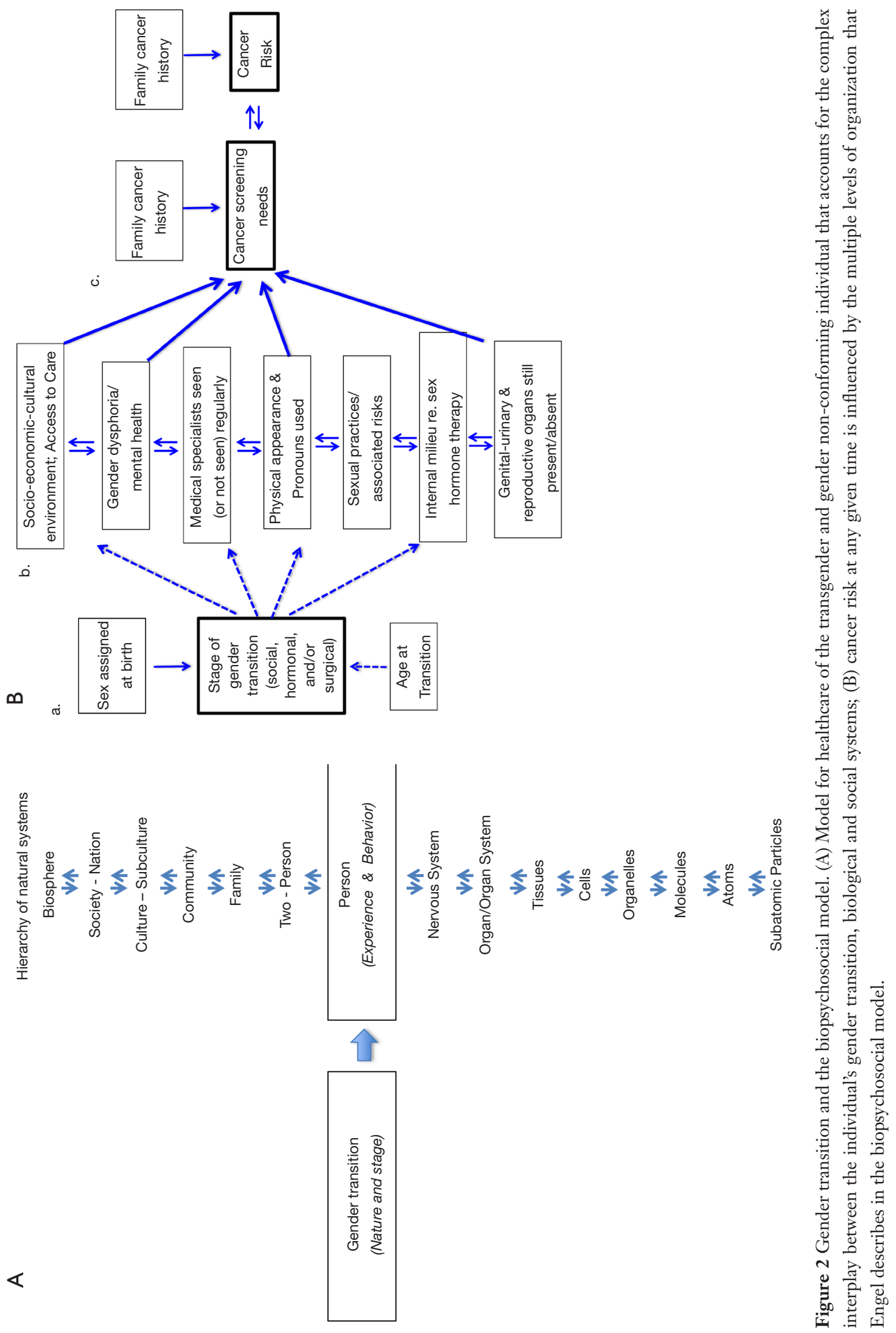


occur during gender transition, and that such changes affect health and illness. In essence, a provider can consider how each subdomain of Engel's biopsychosocial model is affected by the nature, and stage, of gender transition.

We propose a model for healthcare of the transgender and gender non-conforming individual that accounts for the complex interplay between the individual's gender transition, biological and social systems (Figure $2 A$ ).

In the context of cancer screening, the model we propose reminds us that cancer risk at any given time is influenced by the multiple levels of organization that Engel describes in the biopsychosocial model (78), and other factors (Figure $2 B$ ). For example, the age at which an individual commenced transition with use of GAHT, and what stages of transition they have completed, influence factors, which are predictors of cancer screening needs and cancer risk (Figure 2B).

When we consider transgender health from the perspective of the model shown in Figure 2, three key points become clear: first, that gender transition constitutes different changes for different people (i.e., it is highly individual); second, patients can be in different states of transition across different domains at any given time; and third, an individual's present state of gender transition independently influences- and is influenced by, each of the concentric levels of organization within the biological, psychological and social continua.

\section{Conclusions}

The current available evidence does not show that GAHT increases oncologic risk for transgender individuals. However, the available evidence is limited and further research into the effects of long-term GAHT is needed. GAHT does not eliminate the potential for malignancy of the patient's natal sex organs and transgender individuals should undergoing cancer screening for all organs present regardless of transition status. Established guidelines for cisgender individuals and can reasonably applied to transgender patients. Comprehensive cancer screening and prevention initiatives centered on relevant anatomy and high-risk behaviors specific for transgender men and women are needed.

\section{Acknowledgments}

Funding: None.

\section{Footnote}

Reporting Checklist: The authors have completed the PRISMA-ScR reporting checklist. Available at http://dx.doi. org/10.21037/tau-20-954

Peer Review File: Available at http://dx.doi.org/10.21037/ tau-20-954

Conflicts of Interest: Both authors have completed the ICMJE uniform disclosure form (available at http://dx.doi. org/10.21037/tau-20-954). The authors have no conflicts of interest to declare.

Ethical Statement: The authors are accountable for all aspects of the work in ensuring that questions related to the accuracy or integrity of any part of the work are appropriately investigated and resolved.

Open Access Statement: This is an Open Access article distributed in accordance with the Creative Commons Attribution-NonCommercial-NoDerivs 4.0 International License (CC BY-NC-ND 4.0), which permits the noncommercial replication and distribution of the article with the strict proviso that no changes or edits are made and the original work is properly cited (including links to both the formal publication through the relevant DOI and the license). See: https://creativecommons.org/licenses/by-nc-nd/4.0/.

\section{References}

1. Zauber AG. The impact of screening on colorectal cancer mortality and incidence: has it really made a difference? Dig Dis Sci 2015;60:681-91.

2. de Koning HJ, Meza R, Plevritis SK, et al. Benefits and harms of computed tomography lung cancer screening strategies: a comparative modeling study for the U.S. Preventive Services Task Force. Ann Intern Med 2014;160:311-20.

3. Nelson HD, Fu R, Cantor A, et al. Effectiveness of Breast Cancer Screening: Systematic Review and Meta-analysis to Update the 2009 U.S. Preventive Services Task Force Recommendation. Ann Intern Med 2016;164:244-55.

4. Howlander N, Noone A, Krapcho M, et al. SEER cancer statistics review. Bethesda, MD: National Cancer Institute, 2008.

5. Fenton JJ, Weyrich MS, Durbin S, et al. Prostate-Specific 
Antigen-Based Screening for Prostate Cancer: Evidence Report and Systematic Review for the US Preventive Services Task Force. JAMA 2018;319:1914-31.

6. Smith RA, Andrews KS, Brooks D, et al. Cancer screening in the United States, 2017: A review of current American Cancer Society guidelines and current issues in cancer screening. CA Cancer J Clin 2017;67:100-21.

7. World Professional Association for Transgender Health EMRWG. Standards of care for health of transsexual, transgender, and gender nonconforming people, 7th version. 2011. Available online: https://www.wpath. org/media/cms/Documents/SOC\%20v7/SOC\%20V7_ English.pdf

8. Saunders CL, Meads C, Abel GA, et al. Associations Between Sexual Orientation and Overall and Site-Specific Diagnosis of Cancer: Evidence From Two National Patient Surveys in England. J Clin Oncol 2017;35:3654-61.

9. Boehmer U, Miao X, Maxwell NI, et al. Sexual minority population density and incidence of lung, colorectal and female breast cancer in California. BMJ Open 2014;4:e004461.

10. Valanis BG, Bowen DJ, Bassford T, et al. Sexual orientation and health: comparisons in the women's health initiative sample. Arch Fam Med 2000;9:843-53.

11. Kamen C, Blosnich JR, Lytle M, et al. Cigarette Smoking Disparities among Sexual Minority Cancer Survivors. Prev Med Rep 2015;2:283-6.

12. Case P, Austin SB, Hunter DJ, et al. Sexual orientation, health risk factors, and physical functioning in the Nurses' Health Study II. J Womens Health (Larchmt) 2004;13:1033-47.

13. Cathcart-Rake EJ. Cancer in Sexual and Gender Minority Patients: Are We Addressing Their Needs? Curr Oncol Rep 2018;20:85.

14. CDC. CDC-Funded HIV testing: United States, Puerto Rico and the US Virgin Islands. 2015. Available online: http://www.cdc.gov/hiv/library/reports/index.html

15. Clark H, Babu AS, Wiewel EW, et al. Diagnosed HIV Infection in Transgender Adults and Adolescents: Results from the National HIV Surveillance System, 2009-2014. AIDS Behav 2017;21:2774-83.

16. Tabaac AR, Sutter ME, Wall CSJ, et al. Gender Identity Disparities in Cancer Screening Behaviors. Am J Prev Med 2018;54:385-93.

17. Grant J, Mottet L, Tanis J, et al. National transgender discrimination survey report on health and health care, 2010. Available online: https://cancer-network.org/ wpcontent/uploads/2017/02/National_Transgender_
Discrimination_Survey_Report_on_health_and_health_ care.pdf

18. Arcelus J, Bouman WP, Van Den Noortgate W, et al. Systematic review and meta-analysis of prevalence studies in transsexualism. Eur Psychiatry 2015;30:807-15.

19. Kameg BN, Nativio DG. Gender dysphoria in youth: An overview for primary care providers. J Am Assoc Nurse Pract 2018;30:493-8.

20. Cohen-Kettenis PT. Gender identity disorder in DSM? J Am Acad Child Adolesc Psychiatry 2001;40:391.

21. Marshall SA, Allison MK, Stewart MK, et al. Highest Priority Health and Health Care Concerns of Transgender and Nonbinary Individuals in a Southern State. Transgend Health 2018;3:190-200.

22. Reisner SL, Poteat T, Keatley J, et al. Global health burden and needs of transgender populations: a review. Lancet 2016;388:412-36.

23. Olson-Kennedy J, Okonta V, Clark LF, et al. Physiologic Response to Gender-Affirming Hormones Among Transgender Youth. J Adolesc Health 2018;62:397-401.

24. McFarlane T, Zajac JD, Cheung AS. Gender-affirming hormone therapy and the risk of sex hormone-dependent tumours in transgender individuals-A systematic review. Clin Endocrinol (Oxf) 2018;89:700-11.

25. Nelson B. Troubling blind spots in understanding cancer risks among transgender patients: Bias, discrimination, and a lack of good data may be impeding cancer screening and risk assessments for the transgender population. In this article, the second of a 2-part series, we explore the remaining gaps in understanding and communicating the risks. Cancer Cytopathol 2019;127:487-8.

26. Jarin J. The Ob/Gyn and the transgender patient. Curr Opin Obstet Gynecol 2019;31:298-302.

27. Vermeulen MA, Slaets L, Cardoso F, et al. Pathological characterisation of male breast cancer: Results of the EORTC 10085/TBCRC/BIG/NABCG International Male Breast Cancer Program. Eur J Cancer 2017;82:219-27.

28. Chia K, O'Brien M, Brown M, et al. Targeting the androgen receptor in breast cancer. Curr Oncol Rep 2015;17:4.

29. de Blok CJM, Dreijerink KMA, den Heijer M. Cancer Risk in Transgender People. Endocrinol Metab Clin North Am 2019;48:441-52.

30. Gooren LJ, van Trotsenburg MA, Giltay EJ, et al. Breast cancer development in transsexual subjects receiving crosssex hormone treatment. J Sex Med 2013;10:3129-34.

31. Gooren L, Morgentaler A. Prostate cancer incidence 
in orchidectomised male-to-female transsexual persons treated with oestrogens. Andrologia 2014;46:1156-60.

32. Newman PA, Roberts KJ, Masongsong E, et al. Anal Cancer Screening: Barriers and Facilitators Among Ethnically Diverse Gay, Bisexual, Transgender, and Other Men Who Have Sex With Men. J Gay Lesbian Soc Serv 2008;20:328-53.

33. Kobayashi T, Sigel K, Gaisa M. Prevalence of Anal Dysplasia in Human Immunodeficiency Virus-Infected Transgender Women. Sex Transm Dis 2017;44:714-6.

34. Ruanpeng D, Chariyalertsak S, Kaewpoowat Q, et al. Cytological Anal Squamous Intraepithelial Lesions Associated with Anal High-Risk Human Papillomavirus Infections among Men Who Have Sex with Men in Northern Thailand. PLoS One 2016;11:e0156280.

35. Thompson AB, Gillespie SE, Mosunjac MB, et al. Prevalence of Anal Squamous Intraepithelial Lesions in HIV-1-Infected Young Men Who Have Sex With Men and Transwomen. J Low Genit Tract Dis 2018;22:340-7.

36. Olsen CM, Green AC, Nagle CM, et al. Epithelial ovarian cancer: testing the 'androgens hypothesis'. Endocr Relat Cancer 2008;15:1061-8.

37. Grynberg M, Fanchin R, Dubost G, et al. Histology of genital tract and breast tissue after long-term testosterone administration in a female-to-male transsexual population. Reprod Biomed Online 2010;20:553-8.

38. Brown GR, Jones KT. Incidence of breast cancer in a cohort of 5,135 transgender veterans. Breast Cancer Res Treat 2015;149:191-8.

39. Brown GR. Breast Cancer in Transgender Veterans: A Ten-Case Series. LGBT Health 2015;2:77-80.

40. de Blok CJM, Wiepjes CM, Nota NM, et al. Breast cancer risk in transgender people receiving hormone treatment: nationwide cohort study in the Netherlands. BMJ 2019;365:11652.

41. Deutsch MB, Radix A, Wesp L. Breast Cancer Screening, Management, and a Review of Case Study Literature in Transgender Populations. Semin Reprod Med 2017;35:434-41.

42. Kiely D. Transgender Patient Screening: Breast Cancer Risk Assessment and Screening Recommendations. Clin J Oncol Nurs 2017;21:E67-70.

43. Nikolic D, Granic M, Ivanovic N, et al. Breast cancer and its impact in male transsexuals. Breast Cancer Res Treat 2018;171:565-9.

44. Asscheman H, Gooren LJ, Eklund PL. Mortality and morbidity in transsexual patients with cross-gender hormone treatment. Metabolism 1989;38:869-73.
45. James S, Herman J, Rankin S, et al. The report of the 2015 US transgender survey. Available online: http://www. ustranssurvey.org/reports

46. Urban RR, Teng NN, Kapp DS. Gynecologic malignancies in female-to-male transgender patients: the need of original gender surveillance. Am J Obstet Gynecol 2011;204:e9-e12.

47. Grimstad FW, Fowler KG, New EP, et al. Uterine pathology in transmasculine persons on testosterone: a retrospective multicenter case series. Am J Obstet Gynecol 2019;220:257.e1-7.

48. Peitzmeier SM, Khullar K, Reisner SL, et al. Pap test use is lower among female-to-male patients than nontransgender women. Am J Prev Med 2014;47:808-12.

49. Porsch LM, Dayananda I, Dean G. An Exploratory Study of Transgender New Yorkers' Use of Sexual Health Services and Interest in Receiving Services at Planned Parenthood of New York City. Transgend Health 2016;1:231-7.

50. Becasen JS, Denard CL, Mullins MM, et al. Estimating the Prevalence of HIV and Sexual Behaviors Among the US Transgender Population: A Systematic Review and MetaAnalysis, 2006-2017. Am J Public Health 2019;109:e1-8.

51. Brown B, Poteat T, Marg L, et al. Human PapillomavirusRelated Cancer Surveillance, Prevention, and Screening Among Transgender Men and Women: Neglected Populations at High Risk. LGBT Health 2017;4:315-9.

52. Gatos KC. A Literature Review of Cervical Cancer Screening in Transgender Men. Nurs Womens Health 2018;22:52-62.

53. Peitzmeier SM, Reisner SL, Harigopal P, et al. Femaleto-male patients have high prevalence of unsatisfactory Paps compared to non-transgender females: implications for cervical cancer screening. J Gen Intern Med 2014;29:778-84.

54. Potter J, Peitzmeier SM, Bernstein I, et al. Cervical Cancer Screening for Patients on the Female-to-Male Spectrum: a Narrative Review and Guide for Clinicians. J Gen Intern Med 2015;30:1857-64.

55. Baldassarre M, Giannone FA, Foschini MP, et al. Effects of long-term high dose testosterone administration on vaginal epithelium structure and estrogen receptor-alpha and -beta expression of young women. Int J Impot Res 2013;25:172-7.

56. Hembree WC, Cohen-Kettenis P, Delemarre-van de Waal HA, et al. Endocrine treatment of transsexual persons: an Endocrine Society clinical practice guideline. J Clin Endocrinol Metab 2009;94:3132-54. 
57. Wilson M, Berwick DM, DiGuiseppi C. The new edition of the Guide to Clinical Preventive Services. Pediatrics 1996;97:733-5.

58. Harris M, Kondel L, Dorsen C. Pelvic pain in transgender men taking testosterone: Assessing the risk of ovarian cancer. Nurse Pract 2017;42:1-5.

59. Kvach EJ, Hyer JS, Carey JC, et al. Testicular Seminoma in a Transgender Woman: A Case Report. LGBT Health 2019;6:40-2.

60. Wolf-Gould CS, Wolf-Gould CH. A Transgender Woman with Testicular Cancer: A New Twist on an Old Problem. LGBT Health 2016;3:90-5.

61. Huggins C, Hodges CV. Studies on prostatic cancer: I. The effect of castration, of estrogen and of androgen injection on serum phosphatases in metastatic carcinoma of the prostate. 1941. J Urol 2002;168:9-12.

62. King KJ, Nicholson HD, Assinder SJ. Effect of increasing ratio of estrogen: androgen on proliferation of normal human prostate stromal and epithelial cells, and the malignant cell line LNCaP. Prostate 2006;66:105-14.

63. Bosland MC. A perspective on the role of estrogen in hormone-induced prostate carcinogenesis. Cancer Lett 2013;334:28-33.

64. Hu WY, Shi GB, Lam HM, et al. Estrogen-initiated transformation of prostate epithelium derived from normal human prostate stem-progenitor cells. Endocrinology 2011;152:2150-63.

65. Trum HW, Hoebeke P, Gooren LJ. Sex reassignment of transsexual people from a gynecologist's and urologist's perspective. Acta Obstet Gynecol Scand 2015;94:563-7.

66. Coleman E, Bockting W, Botzer M, et al. Standards of Care for the Health of Transsexual, Transgender, and Gender-Nonconforming People, Version 7. Int J Trans 2012;13:165-232.

67. Colebunders B, T'Sjoen G, Weyers S, et al. Hormonal and surgical treatment in trans-women with BRCA1 mutations: a controversial topic. J Sex Med 2014;11:2496-9.

Cite this article as: Sterling J, Garcia MM. Cancer screening in the transgender population: a review of current guidelines, best practices, and a proposed care model. Transl Androl Urol 2020;9(6):2771-2785. doi: 10.21037/tau-20-954
68. Fierz R, Ghisu GP, Fink D. Squamous Carcinoma of the Neovagina after Male-to-Female Reconstruction Surgery: A Case Report and Review of the Literature. Case Rep Obstet Gynecol 2019;2019:4820396.

69. Harder Y, Erni D, Banic A. Squamous cell carcinoma of the penile skin in a neovagina 20 years after male-tofemale reassignment. Br J Plast Surg 2002;5 5:449-51.

70. Fernandes HM, Manolitsas TP, Jobling TW. Carcinoma of the neovagina after male-to-female reassignment. J Low Genit Tract Dis 2014;18:E43-5.

71. Bollo J, Balla A, Rodriguez Luppi C, et al. HPV-related squamous cell carcinoma in a neovagina after male-tofemale gender confirmation surgery. Int J STD AIDS 2018;29:306-8.

72. Yamada K, Shida D, Kato T, et al. Adenocarcinoma arising in sigmoid colon neovagina 53 years after construction. World J Surg Oncol 2018;16:88.

73. Bogliolo S, Gaggero CR, Nadalini C, et al. Long-term risk of malignancy in the neovagina created using colon graft in vaginal agenesis - A case report. J Obstet Gynaecol 2015;35:543-4.

74. Grosse A, Grosse C, Lenggenhager D, et al. Cytology of the neovagina in transgender women and individuals with congenital or acquired absence of a natural vagina. Cytopathology 2017;28:184-91.

75. Engel GL. The need for a new medical model: a challenge for biomedicine. Science 1977;196:129-36.

76. Engel GL. The clinical application of the biopsychosocial model. Am J Psychiatry 1980;137:535-44.

77. Borrell-Carrio F, Suchman AL, Epstein RM. The biopsychosocial model 25 years later: principles, practice, and scientific inquiry. Ann Fam Med 2004;2:576-82.

78. Lehman BJ, David DM, Gruber JA. Rethinking the biopsychosocial model of health: Understanding health as a dynamic system. Social and Personality Psychology Compass 2017;11:e12328. 